\title{
Effects of heat-moisture treatment on molecular interactions and physicochemical properties of tapioca starch
}

\begin{abstract}
Tapioca starch (TS) was heat-moisture treated at 95,110 and $125^{\circ} \mathrm{C}$ with moisture contents of 17, 20 and $23 \%(\mathrm{w} / \mathrm{w})$ for $16 \mathrm{~h}$. Heat-moisture treatment (HMT) increased the apparent amylose content, both crystalline and amorphous areas, and crystallinity of TS. On HMT, gelatinization temperatures were increased but gelatinization enthalpy was decreased; however, both increase and decrease in the gelatinization temperature ranges were observed. Moreover, HMT also increased the retrogradation enthalpy of TS. Total resistant starch (RS) content was decreased but thermostable RS content was increased on HMT. Both pasting characteristics and gel textures of TS were greatly altered by HMT. Specific trends in most physicochemical properties of modified starches were noticed, and were closely correlated with the changes in HMT conditions. Present study showed that HMT-induced structural changes and starch chain interactions exerted a significant effect on X-ray diffraction, thermal properties and RS levels of TS.
\end{abstract}

Keywords: heat-moisture treatment, x-ray diffraction, DSC, resistant starch, gel texture
Volume 3 Issue 3 - 2016

\section{Ya fei Liu, Kalaya Laohasongkram, Saiwarun Chaiwanichsiri}

Department of Food Technology, Faculty of Science, Chulalongkorn University, Thailand

Correspondence: Saiwarun Chaiwanichsiri, Department of Food Technology, Faculty of Science, Chulalongkorn University, Bangkok, Thailand, Tel + 6622I8-55I5-6, Fax + 662254-43I4, Email saiwarun.c@chula.ac.th

Received: October 28, 2016 | Published: December 06, 2016
Abbreviations: TS, tapioca starch; RS, resistant starch; HMT, heat-moisture treatment; AM-L, amylose-lipid; XRD, x-ray diffraction; AM-AM, amylose-amylose; AMP-AMP, amylopectinamylopectin; PV, peak viscosity; CPV, cold paste viscosity; SB, set back; BD, break down; TPA, texture profile analysis; ANOVA, analysis of variance; DMRT, duncan's multiple range test

\section{Introduction}

Cassava (Manihotesculenta Crantz), or tapioca is one of the most important crops grown in Thailand. And, as one of the main production countries, Thailand has gained experience in its starch technology. Tapioca starch (TS) extracted from the roots of cassava plant has been fully studied and widely used in food and industrial applications in Thailand.

TS shows some physicochemical properties superior to most other tuber and the cereal starches, which gives it great preference where specific starch features are desired. For instance, TS possesses a non-cereal, bland taste imparted by the very low lipid content $(<0.1 \%)$, and shows easy cooking, high paste clarity and high paste viscosity. ${ }^{1}$ However, the high breakdown viscosity upon pasting poses a drawback to TS because its cohesive pastes are commonly undesired in food applications. ${ }^{1}$ On account of its lower amylose content and higher molecular weight of amylose, TS pastes retrograde slowly during cooling, which resulted in a lower setback and a lesser noticeable retrogradation behavior., ${ }^{1,2}$ The low setback and slow retro gradation of TS pastes, on one hand, may benefit the texture of long stored starch-based products such as canned foods, but, on the other hand, could restrict its use where excellent gel textures are a prerequisite for specific food processing.
Several modification techniques have been carried out to improve the physicochemical properties of TS so that modified derivatives could serve as potential materials for varied food and non-food applications. ${ }^{3,4}$ Among the ongoing modification methods, starches undergone physical modifications such as hydrothermal treatments (heat-moisture treatment and annealing) are preferable to the chemically treated counterparts due to consumer's concern over the safety of such ingredients. Accordingly, extensive studies have been conducted on heat-moisture treatment (HMT) of starches from various botanical sources, where the effects of HMT on starch physicochemical properties have been examined. ${ }^{5,6}$ HMT could cause starch chain interactions (amylose-amylose, AM-AM and amyloseamylopectin, AM-AMP) and amylose-lipid (AM-L) complexing, which accounted for the increase in starch gelatinization temperatures and also displayed influence on starch retro gradation enthalpy. And HMT-induced starch chain interactions including amylopectinamylopectin (AMP-AMP) have further been shown effects on the levels of rapidly digestible, slowly digestible and resistant starches, ${ }^{8}$ among which resistant starch (RS) has raised great interest due to its physiological benefits. Since molecular interactions of AM-AM, AMP-AMP and AM-AMP on HMT were believed to influence starch thermal properties and thermostable RS levels, one would anticipate the observations of these chain interactions in heat-moisture treated starches by the most likely means of thermal transitions. And it is plausible to assign higher temperatures to the transitions of these chain interactions than to the gelatinization of starch crystallites. Therefore, the objective of present study was to determine the impacts of HMT under varied modification conditions on the physicochemical properties of TS as well as try to verify the presence of HMT-induced starch chain interactions and their effects on resistant starches. 


\section{Materials and methods}

\section{Tapioca starch and heat-moisture treated tapioca starch (HMT-TS)}

Tapioca starch was kindly provided by a local noodle factory (NIT noodle factory, Sukhothai, Thailand). Prior to HMT, exact moisture content was determined and then adjusted to 17,20 and $23 \%$ (w/w, $\mathrm{wb}$ ) by adding the calculated amount of distilled water. Well mixed starches were immediately transferred to food cans and hermetically sealed. After the equilibration at room temperature for $24 \mathrm{~h}$, sealed cans were incubated in hot-air oven at 95,110 and $125^{\circ} \mathrm{C}$ for $16 \mathrm{~h}$. After removing from the oven, cans were unsealed and starch samples were cooled and then dried at $45^{\circ} \mathrm{C}$ till the moisture content reached around $10-12 \%(\mathrm{w} / \mathrm{w})$. Dried samples were ground and sieved though a 120-mesh screen to get heat-moisture treated starches. In this study tapioca starch under specific HMT conditions was termed as HMTTS (moisture content-heating temperature), such as HMT-TS (17-95). Heat-moisture treatment of tapioca starch was duplicated.

\section{Proximate analysis}

Moisture content was determined by the method of AOAC. Apparent amylose content was measured by an iodine colorimetric method. ${ }^{10}$ Chemical analyses were replicated three times.

\section{$X$-ray diffraction characteristics}

X-ray diffraction (XRD) characteristics of starches were obtained by an X-ray diffractometer (Bruker AXS Model D8 Discover, Bruker AXS, USA). Samples were analyzed under the operating conditions as: target voltage $40 \mathrm{kV}$, current $40 \mathrm{~mA}$, increment 0.02 degree/step, scan speed $0.5 \mathrm{sec} / \mathrm{step}$ and a super speed detector (VÅNTEC-1, USA). Data obtained from XRD analysis included crystalline and amorphous areas, crystallinity, inter planar spacings (d) in Angstroms

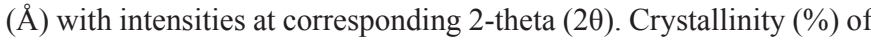
sample was determined by the ratio of crystalline area to the total area of crystalline and amorphous regions.

\section{Thermal properties}

Gelatinization properties of starches were determined by a differential scanning calorimeter (Diamond DSC, Perkin Elmer, Norwalk, Connecticut, USA) equipped with an intercooler unit. Starch $(9 \mathrm{mg})$ and deionized water $(27 \mu \mathrm{l})$ were added to a stainless steel pan and immediately sealed. The sealed pans were equilibrated at room temperature for $2 \mathrm{~h}$ before DSC analysis. Sample pans were then heated from 30 to $150^{\circ} \mathrm{C}$ at a heating rate of $10^{\circ} \mathrm{C} / \mathrm{min}$. An empty pan was used as a reference.

After gelatinization, sample pans were stored at $4^{\circ} \mathrm{C}$ for seven days followed by subsequent equilibration at room temperature for $4 \mathrm{~h}$, and then rescanned by the same profile as gelatinization. Thermal transition onset $\left(\mathrm{T}_{\mathrm{O}}\right)$, peak $\left(\mathrm{T}_{\mathrm{p}}\right)$ and conclusion $\left(\mathrm{T}_{\mathrm{C}}\right)$ temperatures were recorded. The energy (enthalpy, $\Delta \mathrm{H}$ ) required to melt starch crystallites was measured from the areas of endothermic peaks in the thermo grams on a dry weight basis $(\mathrm{J} / \mathrm{g})$, and the temperature range $(\Delta \mathrm{T})$ between onset and conclusion temperatures was calculated as $\mathrm{T}_{\mathrm{C}}-\mathrm{T}_{\mathrm{O}}$. For DSC analysis, each sample was replicated at least twice.

\section{In vitro starch digestibility}

In vitro starch digestibility was expressed as the level of resistant starch (RS) in the starches. Both total and thermostable RS were determined by Megazyme resistant starch assay kit (Megazyme International Ireland, Wicklow, Ireland). The determination of total $\mathrm{RS}$ in starches followed the assay procedure in the kit, and RS content was calculated by the Megazyme Mega-Calc ${ }^{\mathrm{TM}}$ provided along with the assay procedure.

For the determination of thermostable RS, starches were first gelatinized by the method of Chung et al. ${ }^{8}$ Starch $(100 \mathrm{mg})$ and distilled water $(2 \mathrm{ml})$ were added to test tube and capped, and the content was mixed on a vortex mixer for $1 \mathrm{~min}$. The tube was then heated in boiling water for 20 min while gently stirring on a magnetic stirrer with hot plate. After heating, the tube was placed in a water bath at $37^{\circ} \mathrm{C}$ to equilibrate for $10 \mathrm{~min}$. RS determination was then immediately conducted following the above assay procedure. The total and thermostable RS contents of starches were averaged from thee replicates.

\section{Pasting characteristics}

Pasting characteristics of starches were determined by a Rapid Visco-Analyzer (RVA; Series S4A, Newport Scientific, Australia). Starch $(3.0 \mathrm{~g}, 12 \%$ moisture basis) was weighed directly in the RVA canister and distilled water was added to obtain a suspension with total weight of $28.0 \mathrm{~g}$. The suspension was heated and cooled by the procedure of the AACC ${ }^{11}$ standard method No. 61-02.01. Parameters of pasting characteristics included peak viscosity (PV), the highest viscosity during heating; hot paste viscosity (HPV) at the end of holding at $95^{\circ} \mathrm{C}$; cold paste viscosity $(\mathrm{CPV})$ at the end of holding at $50^{\circ} \mathrm{C}$; breakdown $(\mathrm{BD}=\mathrm{PV}-\mathrm{HPV})$; setback $(\mathrm{SB}=\mathrm{CPV}-\mathrm{HPV})$ and pasting temperature $\left(\mathrm{P}_{\mathrm{T}}\right)$, the temperature where great increase in viscosity were observed. All measurements were processed in duplicate.

\section{Gel texture}

A TA-XTi2 texture analyzer (Stable Micro Systems, Godalming, UK) was used to determine the texture of gels formed in the RVA canisters after pasting test. ${ }^{12}$ After removal of RVA paddles, canisters were covered with Para film ${ }^{\square}$, and stored at $4^{\circ} \mathrm{C}$ overnight. Sample was then equilibrated at room temperature for $4 \mathrm{~h}$ before texture profile analysis (TPA). TPA of the gels was conducted using a $6.0 \mathrm{~mm}$ diameter cylindrical probe to puncture $10.0 \mathrm{~mm}$ depth of the gel at $1.0 \mathrm{~mm} / \mathrm{s}$. The pause between first and second cycles was $5 \mathrm{~s}$. Gel TPA parameters of hardness, adhesiveness, springiness, cohesiveness and gumminess were recorded. Each gel was tested at five different positions on the surface, and the result was obtained from all the data for each sample.

\section{Statistical analysis}

All the mean values and standard deviations were analyzed by SPSS v. 16. Analysis of variance (ANOVA) was performed for multiple treatments and the differences were reported at a confidence level of $95 \%(P \leq 0.05)$ by Duncan's multiple range test (DMRT).

\section{Results and discussion}

\section{Impact of HMT on apparent amylose content}

Apparent amylose contents (AAC) of native and modified tapioca starches are shown in Figure 1. HMT increased the AAC of TS, and HMT at $110^{\circ} \mathrm{Cproduced}$ the highest values followed by those at $95^{\circ} \mathrm{C}$ and $125^{\circ} \mathrm{C}$ under each moisture level. Moreover, at each heating temperature, the HMT with $20 \%$ moisture resulted in 
the highest values followed by those with $23 \%$ and $17 \%$ moisture. The highest AAC was obtained by the mid-point HMT condition (i.e. $110^{\circ} \mathrm{C}$ and $20 \%$ moisture) among all the treatments; however, similar observations have not been reported. Lu et al. ${ }^{13}$ reported that HMT could cause thermal degradation of the exterior linear chains of amylopectin. And these degraded exterior linear chains became just like amylose chains and would complex with other amylose chains or lipids, responsible for the increase in amylose content after HMT. ${ }^{14}$ While HMT at higher heating temperatures (or moisture levels) might cause higher degrees of amylopectin degradation, HMT-induced starch chain interactions were severer at $125^{\circ} \mathrm{C}$ (or $23 \%$ moisture) than at $110^{\circ} \mathrm{C}$ (or $20 \%$ moisture) and $95^{\circ} \mathrm{C}$ (or $17 \%$ moisture). On AAC determination, newly created amylose could be counteracted by the amylose interactions with amylose or amylopectin from more severe HMT conditions, which might explain why the mid-point condition constantly showed the higher AAC.

\section{Impact of HMT on X-ray diffraction characteristics}

XRD characteristics of native and modified tapioca starches are presented in Table 1. For all the starch samples, thee noticeable diffraction peaks at $\sim 15,17$ and $23^{\circ} 2 \theta$ were observed. They typified the XRD pattern of cereal starches and therefore indicated an A-pattern of TS and HMT-TS. Although HMT did not change the XRD pattern of TS, HMT-TS demonstrated higher crystalline and amorphous areas as well as higher crystallinity (Table 1 ). Chung et al. ${ }^{8}$ reported that HMT might increase the mobility of double helices in the crystalline regions causing disruption of linking hydrogen bonds. And this might contribute to the increase in amorphous areas. Crystallite perfection, due to the HMT-induced rearrangement of double helices within the crystalline regions, could form more ordered crystalline arrays ${ }^{7,15,16}$ which possibly accounted for the larger crystalline areas of HMT-TS. Regarding the starch crystallinity, it has been influenced by several factors including crystallite size, double helices array within the crystallite, average chain length of amylopectin and the amount of short chain fraction of amylopectin. ${ }^{17}$

Moreover, the comparison of peak intensities between TS and HMT-TS showed that HMT increased the intensities of major peaks, but also caused the disappearance of some characteristic peaks (Table 1). Hoover et al. ${ }^{7}$ have reported such impact of HMT on shifting and causing loss of existing d-spacings of major diffraction peaks of cereal, legume, and tuber starches. The above authors attributed these changes to the thermal energy and moisture on HMT which rearranged the double helices or caused the rupture of linkages between adjacent double helices.

Table I X-RAY diffraction characteristics of native and heat-moisture treated tapioca starches

\begin{tabular}{|c|c|c|c|c|c|c|c|c|}
\hline \multirow{2}{*}{ Sample } & \multicolumn{4}{|c|}{ Interplanar spacings (d) in $\AA$ with intensities (CPS) ${ }^{1}$} & \multicolumn{4}{|c|}{ X-Ray diffraction pattern and crystallinity } \\
\hline & $15.1^{\circ} 2 \theta$ & $16.9^{\circ} 2 \theta$ & $17.2^{\circ} 2 \theta$ & $23^{\circ} 2 \theta$ & X-ray pattern & $\begin{array}{l}\text { Crystalline } \\
\text { area }\end{array}$ & $\begin{array}{l}\text { Amorphous } \\
\text { area }\end{array}$ & Crystallinity (\%) \\
\hline TS & $5.86(1142)$ & $5.21(1257)$ & $5.15(1281)$ & $3.84(1002)$ & $\mathrm{A}$ & 3769 & 12777 & 22.78 \\
\hline HMT-TS (17-95) & $5.84(1418)$ & $5.23(1486)$ & $5.19(1553)$ & $3.87(1274)$ & $\mathrm{A}$ & 4882 & 14954 & 24.61 \\
\hline HMT-TS (17-110) & $5.86(1406)$ & $5.26(1528)$ & $5.20(1531)$ & $3.88(1217)$ & $\mathrm{A}$ & 4639 & 14654 & 24.05 \\
\hline HMT-TS (17-125) & $5.86(2461)$ & $5.22(2623)$ & $5.18(2708)$ & $3.86(2125)$ & A & 8634 & 23227 & 27.1 \\
\hline HMT-TS (20-95) & $5.87(2484)$ & $5.23(2701)$ & $5.19(2719)$ & $3.86(2128)$ & A & 8756 & 23737 & 26.95 \\
\hline HMT-TS (20-110) & $5.88(2400)$ & $5.22(2614)$ & $5.18(2607)$ & $3.87(1989)$ & A & 8136 & 23038 & 26.1 \\
\hline HMT-TS (20-125) & $5.83(1253)$ & $\mathrm{ND}^{2}$ & $5.15(1377)$ & $3.86(1065)$ & A & 4033 & 13442 & 23.08 \\
\hline HMT-TS (23-95) & $5.87(2437)$ & ND & $5.19(2663)$ & $3.86(2112)$ & $\mathrm{A}$ & 8274 & 23318 & 26.19 \\
\hline HMT-TS (23-110) & $5.88(2189)$ & $5.23(2343)$ & $5.19(2389)$ & $3.88(1898)$ & A & 7258 & 21555 & 25.19 \\
\hline HMT-TS (23-125) & $5.87(2403)$ & $5.21(2523)$ & $5.17(2538)$ & $3.86(1994)$ & A & 7743 & 22612 & 25.51 \\
\hline
\end{tabular}

CPS, counts per second; ND, not detected

\section{Impact of HMT on thermal properties}

Upon gelatinization, the endothermic transitions of HMT-TS showed a shift toward higher temperatures in comparison to that of TS (Figure 2). At each moisture level, the increase in the heating temperatures of HMT was accompanied by the elevated gelatinization temperatures, and vice versa (Figure 3). This indicated that both high temperature and high moisture used in HMT promoted the recrystallization of starch granules. It has been reported that HMT could increase the gelatinization temperatures of starches. ${ }^{7,15,16,18}$ These authors claimed that HMT-induced starch chain interactions could restrict the swelling and mobility of starch chains in the amorphous regions causing higher temperatures for crystallite melting. Moreover, the aforementioned thermal degradation of weak structures of amylopectin and the crystallite perfection on HMT may further enhance the thermal stability of starch crystallites contributing to higher gelatinization temperatures.

From Figure 3, in comparison with TS, HMT-TS showed decreased gelatinization enthalpy $\left(\Delta \mathrm{H}_{\mathrm{G}}\right)$. Furthermore, trends were observed between the changes in HMT conditions and the decrease in $\Delta \mathrm{H}_{\mathrm{G}}$ of HMT-TS: increase in the moisture levels and/or heating temperatures of HMT was followed by the progressive decrease in $\Delta \mathrm{H}_{\mathrm{G}}$ (Figure $3)$. This implied that more penetration of water molecules into starch granules would cause more disruption of double helices resulting in less energy required to melt the remaining crystallites. According to the report of Hoover et al., ${ }^{7}$ increased moisture level of HMT would facilitate the movement of double-helical chains promoting 
the disruption of double helices. Double helices in both crystalline and amorphous regions could be disrupted by HMT, which led to less amount of double helices unraveling and melting during starch gelatinization and the decreased $\Delta \mathrm{H}_{\mathrm{G}}{ }^{17}$ Cooke et al. ${ }^{19}$ reported, further verified by Hoover et al., ${ }^{7}$ that $\Delta \mathrm{H}_{\mathrm{G}}$ primarily reflected the loss of double helical order rather than the crystalline structure. In our study, HMT-TS, with higher crystallinity than TS, showing smaller $\Delta \mathrm{H}_{\mathrm{G}}$ was due to the lesser disruption of double helices during gelatinization.

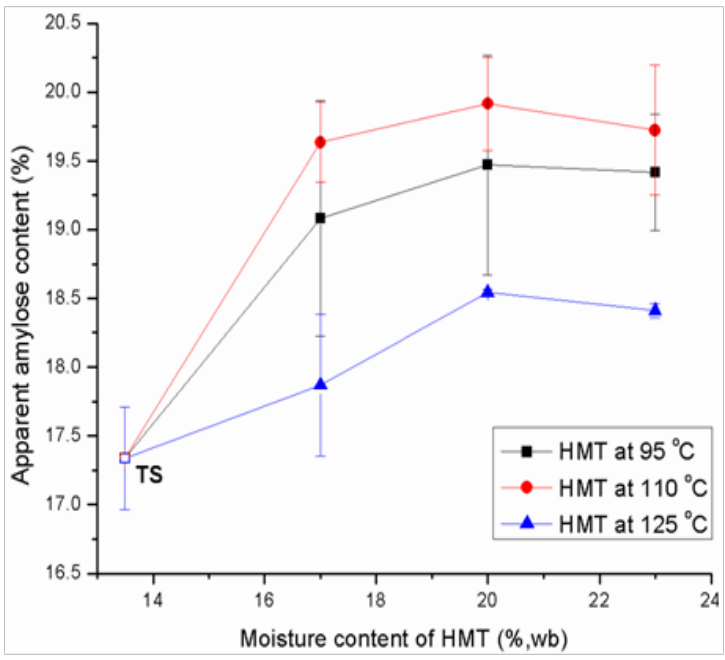

Figure 1 Apparent amylose content of the ( $\square$ ) native tapioca starch (TS) and $(\bullet, \mathbf{\square}, \mathbf{\Delta})$ those that were heat-moisture treated (HMT-TS) at $(\boldsymbol{\Delta}) 95^{\circ} \mathrm{C},(\boldsymbol{\bullet})$ $110^{\circ} \mathrm{C}$ or $(\bullet) 125^{\circ} \mathrm{C}$ with different moisture contents. Data shown are derived from two replications of experiment.

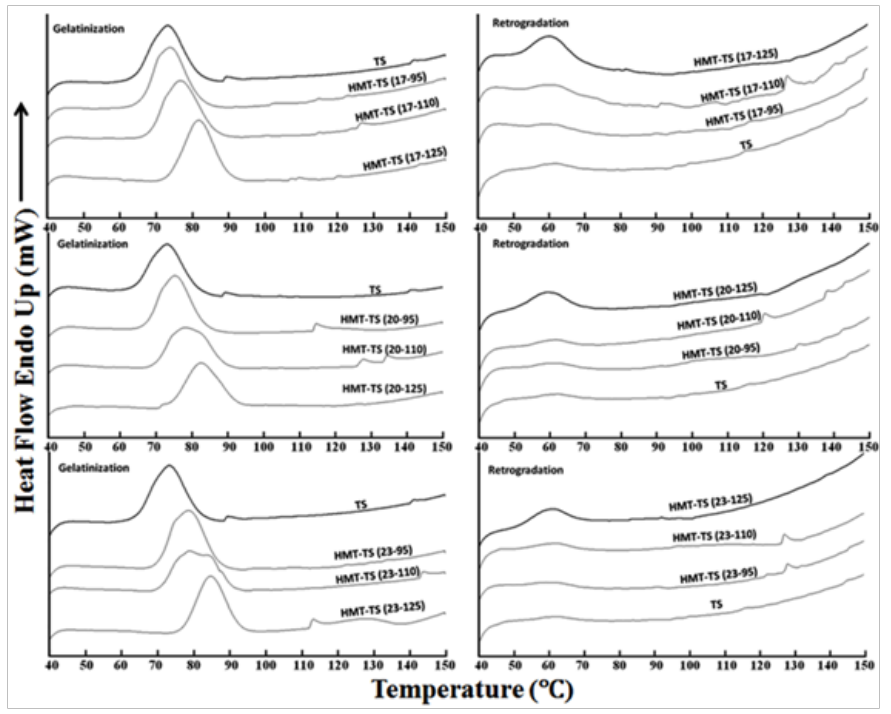

Figure 2 DSC thermograms showing the gelatinization and retro gradation of the native tapioca starch (TS) and those that were heat-moisture treated (HMT-TS). Data shown are derived from two replications of experiment.

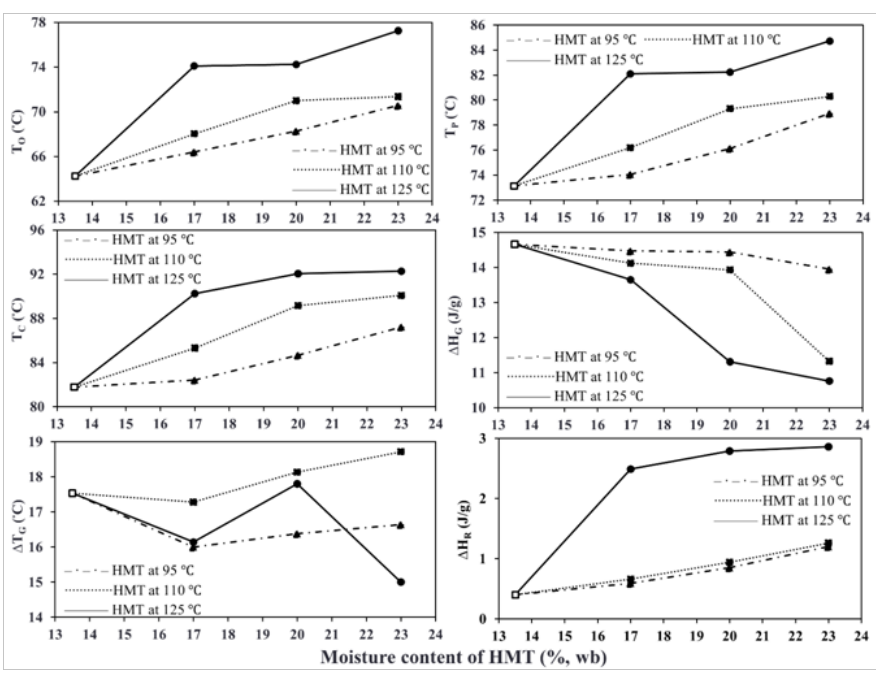

Figure 3 DSC thermal properties of the ( $\square$ ) native tapioca starch (TS) and $(\bullet, \mathbf{\Lambda})$ those that were heat treated (HMT-TS) at $(\mathbf{\Lambda}) 95^{\circ} \mathrm{C},(\mathbf{-}) 110^{\circ} \mathrm{C}$ or $(\bullet) 125^{\circ} \mathrm{C}$. Thermal properties are shown as the thermal onset (To), peak (Tp) and conclusion (Tc) temperatures, and changes in the level of the starch gelatinization enthalpy $(\square \mathrm{HG})$, gelatinization temperature $(\square \mathrm{TG})$ and amylopectin retro gradation enthalpy $(\square \mathrm{H})$. Data shown are derived from two or more replications.

Regarding the gelatinization temperature range $\left(\Delta \mathrm{T}_{\mathrm{G}}\right)$, HMT caused both broadened and narrowed $\Delta \mathrm{T}_{\mathrm{G}}$ of TS (Figure 3 ). The $\Delta \mathrm{T}_{\mathrm{G}}$ has been interpreted as the indication of homogeneity of ordered structures inside starch granules. ${ }^{20}$ Differences in the $\Delta T_{G}$ among HMT-TS suggested varied homogeneity of crystallites within the starch granules. ${ }^{17}$ Starch with increased $\Delta T_{G}$ after HMT has been reported by other researchers. ${ }^{7,15-18}$ Starch with decreased $\Delta \mathrm{T}_{\mathrm{G}}$ after HMT, however, could be due to the modifications under some conditions benefiting the development of more homogeneous crystallites. Similar results of HMT causing decrease in $\Delta \mathrm{T}_{\mathrm{G}}$ were also observed for rice starch. ${ }^{21}$

Within the higher temperature range $\left(>95^{\circ} \mathrm{C}\right)$ on the DSC traces, HMT-TS showed some multiphasic endothermic transitions (Figure 2). The lipid content of tapioca starch is known to be very low, which could not contribute to the complexation of amylose and lipid. Therefore, these minor but noticeable melting peaks might be the disordering of newly formed structures (i.e. HMT-induced starch chain interactions) instead of AM-L complexes. Co-crystallization between amylose and amylopectin could possibly occur on HMT. ${ }^{18}$ Regarding the thermal stabilities of these HMT-induced starch chain interactions, AM-AM was reported to be much stronger than AMAMP and AMP-AMP. ${ }^{22}$ The phase transition of recrystallized amylose has been shown in the temperature range of $110-150^{\circ} \mathrm{C} .{ }^{18}$ Therefore, these minor endothermic transitions might be the dissociation of crystallized starch chains that had different thermal stabilities due to varied degrees of chain association on HMT.

Retro gradation of TS showed minute endothermic transition in the DSC thermogram (Figure2). In contrast, HMT-TS displayed distinguishable melting peaks expressed by their larger endothermic peak areas than that of TS (Figure 2). Indicated by the endothermic peak areas, retro gradation enthalpy $(\Delta \mathrm{H})$ of HMT-TS was influenced by the conditions of HMT. It is obvious in Figure 3 that at constant 
heating temperature, higher moisture level of HMT resulted in higher $\Delta \mathrm{H}$, and vice versa. Furthermore, at each moisture level, HMT at $125^{\circ} \mathrm{C}$ produced much higher $\Delta \mathrm{H}$ than both at 95 and $110^{\circ} \mathrm{C}$ (Figure $3)$. Starch retro gradation enthalpy $(\Delta \mathrm{H})$ actually measures the melting of recrystallized amylopectin, which is accordingly relevant to the process of amylopectin recrystallization. The very low $\Delta \mathrm{H}$ of TS was due to the long unit chain length of amylopectin demanding extended period of time to recrystallize..$^{23}$ Increased $\Delta H$ of HMT-TS indicated that HMT promoted the recrystallization process of amylopectin, and the same effect of HMT on facilitating starch retro gradation has also been reported. ${ }^{13,14}$ From the results of starch X-ray diffraction, the higher peak intensity of HMT-TS indicated a rearranged amylopectin chain array, which would expedite the association of amylopectin chains during starch retro gradation. And therefore highly recrystallized amylopectin melted on reheating would produce larger $\Delta \mathrm{H}$ of HMT-TS than TS.

\section{Impact of HMT on resistant starch content}

Noticed from Figure 4 that TS had a total RS content higher than those of HMT-TS, but a thermostable RS content lower than those of HMT-TS. And significant differences in the thermostable RS content were found between TS and HMT-TS from more severe modifications. Moreover, the thermostable RS contents of HMT-TS increased with an increase in the heating temperature at each moisture level. Reported by Englyst et al., ${ }^{24} \mathrm{RS}$ derived from granular starches belonged to the second type RS (RS 2), and their resistance to enzymatic hydrolysis depended on the granular morphology and crystalline organization. ${ }^{25}$ The high total RS content of TS suggested a low susceptibility toward enzymatic hydrolysis, which might be due to the fact that TS granules possessed multiple layers and a tightly organized structure causing less accessibility to hydrolyzing enzymes. ${ }^{2}$ Compared with TS, the lower and varying total RS contents of HMT-TS might be ascribed to the HMT-induced morphological alterations of starch granules. ${ }^{26}$ Granular damage such as concave surface and holes caused by HMT would result in weaker resistance of starch granules to enzymatic hydrolysis. ${ }^{14}$ Similarly, decrease in the total RS contents of maize starches on HMT has been reported. ${ }^{8,27}$

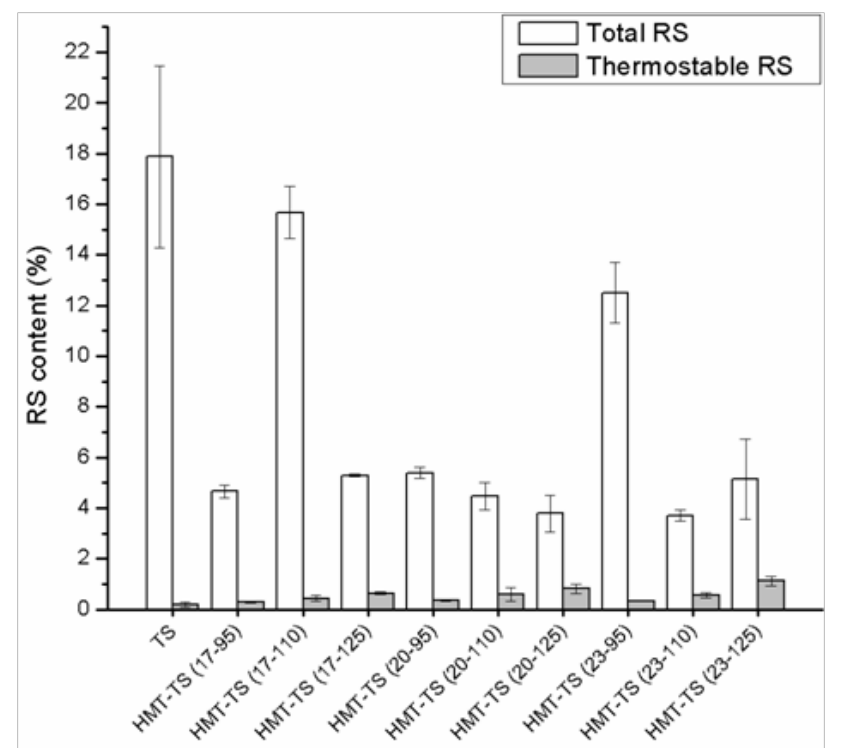

Figure 4 Resistant starch (RS) contents thermostable RS, of native and heat-moisture treated tapioca
For all starch samples, thermostable RS contents were much lower than total RS contents due to starch gelatinization. It disrupted the barrier of granular structure and exposed amorphous and crystalline areas to amylolytic enzymes causing sufficient hydrolysis. The HMTTS exhibited higher thermostable RS contents than TS, which could be due to the HMT-induced starch chain interactions: AM-AM, AMPAMP and AM-AMP were believed to survive starch gelatinization and further restrain the accessibility of starch chains to hydrolyzing enzymes. ${ }^{22}$ Moreover, the aforementioned correlation between HMT conditions and the thermostable RS contents of HMT-TS suggested that HMT-induced starch chain interactions with higher thermal stabilities would result in higher resistance to enzyme hydrolysis. In agreement, HMT has caused increase in the thermostable RS contents of corn, pea and lentil starches ${ }^{22}$ and the boiling-stable RS contents of maize starch. ${ }^{27}$

\section{Impact of HMT on pasting characteristics}

The RVA pasting characteristics of all starch samples were presented in Table 2. Compared with TS, HMT-TS showed decreased $\mathrm{PV}, \mathrm{BD}$ and $\mathrm{SB}$, but increased $\mathrm{P}_{\mathrm{T}}$. However, the HPV and CPV of HMT-TS showed to be higher or lower than those of TS, and varied greatly along the HMT conditions. Regarding the PV, HPV, BD, CPV and SB, HMT-TS showed these characteristics in the same trend: at constant moisture level, increased heating temperature of HMT was followed by a decrease in these viscosity parameters (Table 2). Whereas, the $\mathrm{P}_{\mathrm{T}}$ of HMT-TS displayed an opposite trend: at constant moisture level, increased heating temperature of HMT was followed by an increase in the $\mathrm{P}_{\mathrm{T}}$ (Table 2).

PV of TS was decreased by HMT, which was probably due to the HMT-induced morphological alterations and crystalline disruptions of starch granules. At constant moisture level, increase in the heating temperature might cause more severe granular damage, thus, resulting in weaker resistance to granule swelling. Such influence was also revealed by the variations in the HPV of HMT-TS. Under low heating temperature (i.e. $95^{\circ} \mathrm{C}$ ) the starch granular rigidity still remained high on HMT, which would produce higher resistance to the shearing and then higher HPV. Breakdown (BD), viscosity change from PV to HPV, reflects the thermal stability of swollen starch granules. Compared with TS, HMT-TS showed lower BD suggesting higher thermal stability of the starch granules, which was most likely due to the HMT-induced starch chain interactions. CPV is indicative of the recrystallization of solubilized amylose upon cooling, ${ }^{28}$ which results in an increase in the paste viscosity. And the viscosity change from $\mathrm{HPV}$ to $\mathrm{CPV}$ is denoted as SB that indicates the extent of starch retro gradation. The differences in both CPV and SB between TS and HMTTS suggested that HMT had influence on starch amylose leaching and retro gradation during pasting test. Furthermore, note that under varied HMT conditions changes in $\mathrm{P}_{\mathrm{T}}$ followed the same trends as that in gelatinization temperatures, which implied consistency of temperature changes between starch gelatinization and pasting on HMT. Reportedly, the general effects of HMT on the pasting properties of tapioca starches were suppression in PV, increase or decrease in $\mathrm{CPV}$ and an increase in $\mathrm{P}_{\mathrm{T}},{ }^{3,29}$ and our results were in agreement with these observations.

\section{Impact of HMT on gel texture}

Gel TPA results of TS and HMT-TS are listed in Table 3. The prominent texture characteristic of TS gel was the absence of adhesiveness, springiness, cohesiveness and gumminess. Whereas, 
HMT-TS showed all the TPA parameters, and differences were noticed among them due to the varied HMT conditions. Gel hardness is commonly used to evaluate the texture contribution of starches in food applications. Gel hardness of TS was significantly higher than those of HMT-TS. The HMT-TS showed gel hardness in a specific trend relevant to the HMT conditions: at each moisture level, HMT at $125^{\circ} \mathrm{C}$ resulted in the highest gel hardness followed by those at 95 and $110^{\circ} \mathrm{C}$.

Under puncture test, the absence of adhesiveness, springiness, cohesiveness and gumminess of TS gel implied that the probe failed to break and penetrate into the gel. This was evidenced by the fact that gel adhesiveness, defined as the negative area in the first cycle, did not appear in the TPA graph of TS gel. Since the TPA parameters are measured based on a typical two-cycle TPA-style result graph, the lack of adhesiveness would then cause the absence of other TPA parameters. While the hardness of HMT-TS gels was obtained from TPA tests where the gels were punctured, then the "hardness" of TS gel should be interpreted as compressibility. Although both setback (SB) viscosity and gel hardness of HMT-TS were smaller than those of TS, inconsistency was observed between the trends of SB and gel hardness of HMT-TS. In pasting test, SB mainly reflected the retro gradation of leached amylose during short time cooling. Whereas, starch gel hardness was influenced by the complex interplay of continuous and dispersed phases of gelatinized starch and the crystallization of amylopectin and leached amylose during storage. ${ }^{30}$

Table 2 RVA pasting characteristics of native and heat-moisture treated tapioca starches

\begin{tabular}{|c|c|c|c|c|c|c|}
\hline \multirow{2}{*}{ Sample } & \multicolumn{6}{|c|}{ RVA Pasting Characteristics ${ }^{1)}$} \\
\hline & PV (RVU) & HPV (RVU) & BD (RVU) & CPV (RVU) & SB (RVU) & $\mathbf{P}_{\mathrm{T}}\left({ }^{\circ} \mathrm{C}\right)$ \\
\hline TS & $399.42 \pm 1.13 \mathrm{a}$ & $156.73 \pm 0.79 b$ & $242.69 \pm 0.76 \mathrm{a}$ & $234.73 \pm 4.33 b$ & $78.00 \pm 5.03 \mathrm{a}$ & $69.44 \pm 0.35 h$ \\
\hline HMT-TS (17-95) & $355.21 \pm 28.64 b$ & $169.21 \pm 3.85 \mathrm{a}$ & $186.00 \pm 24.83 b$ & $227.44 \pm 3.59 \mathrm{~b}$ & $58.23 \pm 0.86 \mathrm{~b}$ & $70.95 \pm 0.73 \mathrm{~g}$ \\
\hline HMT-TS (17-110) & $191.48 \pm 19.56 \mathrm{~d}$ & $100.64 \pm 5.42 \mathrm{c}$ & $90.83 \pm 14.15 \mathrm{e}$ & $141.42 \pm 10.20 \mathrm{c}$ & $40.77 \pm 4.79 \mathrm{c}$ & $72.00 \pm 0.52 \mathrm{f}$ \\
\hline HMT-TS (17-125) & $20.29 \pm 3.21 \mathrm{~g}$ & $9.94 \pm 1.63 \mathrm{e}$ & $10.35 \pm 1.62 \mathrm{~g}$ & $14.06 \pm 1.91 \mathrm{e}$ & $4.13 \pm 0.40 \mathrm{e}$ & $81.64 \pm 0.48 \mathrm{c}$ \\
\hline HMT-TS (20-95) & $318.60 \pm 28.31 \mathrm{c}$ & $168.23 \pm 7.93 \mathrm{a}$ & $150.38 \pm 20.40 \mathrm{c}$ & $233.04 \pm 1.98 b$ & $64.82 \pm 9.39 b$ & $71.76 \pm 0.69 f$ \\
\hline HMT-TS (20-110) & $134.44 \pm 3.06 \mathrm{f}$ & $81.54 \pm 2.64 d$ & $52.90 \pm 0.44 \mathrm{f}$ & $114.27 \pm 2.28 \mathrm{~d}$ & $32.73 \pm 0.41 \mathrm{~d}$ & $74.64 \pm 0.39 \mathrm{e}$ \\
\hline HMT-TS (20-125) & $22.02 \pm 3.09 \mathrm{~g}$ & $12.07 \pm 1.22 \mathrm{e}$ & $9.95 \pm 1.92 \mathrm{~g}$ & $17.70 \pm 1.44 \mathrm{e}$ & $5.63 \pm 0.30 \mathrm{e}$ & $82.58 \pm 0.22 b$ \\
\hline HMT-TS (23-95) & $304.41 \pm 22.60 \mathrm{c}$ & $172.64 \pm 7.67 \mathrm{a}$ & $131.77 \pm 14.98 \mathrm{~d}$ & $246.88 \pm 1.97 \mathrm{a}$ & $74.23 \pm 9.52 \mathrm{a}$ & $74.24 \pm 0.41 \mathrm{e}$ \\
\hline HMT-TS (23-110) & $166.23 \pm 19.80 \mathrm{e}$ & $104.34 \pm 11.56 \mathrm{c}$ & $61.90 \pm 8.27 f$ & $150.27 \pm 16.53 \mathrm{c}$ & $45.93 \pm 4.99 \mathrm{c}$ & $75.55 \pm 0.61 d$ \\
\hline HMT-TS (23-125) & $12.09 \pm 1.20 \mathrm{~g}$ & $6.96 \pm 1.05 \mathrm{e}$ & $5.13 \pm 0.20 \mathrm{~g}$ & $11.08 \pm 1.24 \mathrm{e}$ & $4.12 \pm 0.38 \mathrm{e}$ & $83.52 \pm 0.34 \mathrm{a}$ \\
\hline
\end{tabular}

I)PV: Peak Viscosity; HPV, hot paste viscosity; BD, break down; CPV, cold paste viscosity; SB, set back; PT, pasting temperature Means of each characteristic followed by different letters in the same column are significantly different at $P<0.05$.

Table 3 Gel texture profile analysis (TPA) of native and heat-moisture treated tapioca starches.

\begin{tabular}{llllll}
\hline \multirow{2}{*}{ Sample } & \multicolumn{4}{l}{ Texture Profile Analysis (TPA) Characteristics } & \\
\cline { 2 - 5 } & $\begin{array}{l}\text { Hardness } \\
\text { (g force) }\end{array}$ & $\begin{array}{l}\text { Adhesiveness } \\
(\mathbf{g} \cdot \mathbf{s e c})\end{array}$ & Springiness & Cohesiveness & $\begin{array}{l}\text { Gumminess } \\
\text { (g force) }\end{array}$ \\
\hline TS & $24.52 \pm 1.15 \mathrm{a}$ & $\mathrm{ND}^{1)}$ & $0.00 \pm 0.00 \mathrm{~d}$ & $0.00 \pm 0.00 \mathrm{f}$ & $0.00 \pm 0.00 \mathrm{~h}$ \\
HMT-TS (17-95) & $16.27 \pm 0.81 \mathrm{~d}$ & $13.23 \pm 3.70 \mathrm{f}$ & $0.89 \pm 0.02 \mathrm{c}$ & $0.44 \pm 0.02 \mathrm{e}$ & $7.14 \pm 0.23 \mathrm{~d}$ \\
HMT-TS (17-110) & $10.71 \pm 0.54 \mathrm{j}$ & $32.68 \pm 1.68 \mathrm{c}$ & $0.92 \pm 0.01 \mathrm{~b}$ & $0.56 \pm 0.03 \mathrm{~b}$ & $5.98 \pm 0.19 \mathrm{~g}$ \\
HMT-TS (17-125) & $17.47 \pm 0.70 \mathrm{c}$ & $44.66 \pm 9.10 \mathrm{a}$ & $0.93 \pm 0.02 \mathrm{ab}$ & $0.58 \pm 0.01 \mathrm{ab}$ & $9.75 \pm 0.22 \mathrm{~b}$ \\
HMT-TS (20-95) & $14.87 \pm 0.39 \mathrm{f}$ & $18.10 \pm 6.44 \mathrm{e}$ & $0.90 \pm 0.02 \mathrm{c}$ & $0.46 \pm 0.05 \mathrm{~d}$ & $6.91 \pm 0.45 \mathrm{def}$ \\
HMT-TS (20-110) & $11.44 \pm 0.66 \mathrm{i}$ & $39.52 \pm 1.35 \mathrm{~b}$ & $0.93 \pm 0.01 \mathrm{~b}$ & $0.59 \pm 0.02 \mathrm{a}$ & $6.80 \pm 0.39 \mathrm{f}$ \\
HMT-TS (20-125) & $19.05 \pm 0.92 \mathrm{~b}$ & $40.23 \pm 7.35 \mathrm{~b}$ & $0.94 \pm 0.02 \mathrm{a}$ & $0.56 \pm 0.02 \mathrm{~b}$ & $10.73 \pm 0.51 \mathrm{a}$ \\
HMT-TS (23-95) & $13.48 \pm 0.46 \mathrm{~g}$ & $28.24 \pm 5.13 \mathrm{~d}$ & $0.90 \pm 0.02 \mathrm{c}$ & $0.51 \pm 0.03 \mathrm{c}$ & $6.83 \pm 0.32 \mathrm{ef}$ \\
HMT-TS (23-110) & $11.95 \pm 0.65 \mathrm{~h}$ & $40.66 \pm 1.72 \mathrm{~b}$ & $0.92 \pm 0.01 \mathrm{~b}$ & $0.59 \pm 0.01 \mathrm{a}$ & $7.08 \pm 0.40 \mathrm{de}$ \\
HMT-TS (23-125) & $15.46 \pm 0.42 \mathrm{e}$ & $39.15 \pm 3.90 \mathrm{~b}$ & $0.94 \pm 0.03 \mathrm{a}$ & $0.58 \pm 0.02 \mathrm{ab}$ & $8.92 \pm 0.43 \mathrm{c}$ \\
\hline
\end{tabular}

1) N, not detected

Means of each characteristic followed by different letters in the same column are significantly different at $\mathrm{P}<0.05$. 


\section{Conclusion}

Changes in some physicochemical properties of tapioca starch were observed on heat-moisture treatment. HMT-induced crystalline disruption and starch chain interactions were the two major underlying causes of these changes. Although the A-pattern was not changed by HMT, crystalline disruption and rearrangement of double helices showed impact on the crystalline and amorphous areas as well as the crystallinity. The disruption of linking hydrogen bonds impaired the integrity of double helices resulting in decreased gelatinization enthalpy, while starch chain interactions that increased the gelatinization temperatures were observed in higher temperature areas. These starch chain interactions also played an important role in the formation of thermostable resistant starches. Furthermore, specific trends in the apparent amylose content, thermal properties, resistant starch content, pasting characteristics and gel TPA of HMT-TS were noticed and followed the changes in the moisture levels and heating temperatures of HMT. Accordingly, the goal of tailor-made HMT-TS with desired features could be obtained by HMT of TS under proper conditions for food and non-food applications.

\section{Acknowledgements}

The financial support for this study from the Graduate School of Chulalongkorn University is gratefully acknowledged. The authors also wish to thank the NIT noodle factory for providing the raw materials.

\section{Conflict of interest}

The author declares no conflict of interest.

\section{References}

1. Moorthy SN. Tropical sources of starch. In: Eliasson AC editor. Starch in Food - Structure, Function and applications. Boca Raton, USA; 2004. p. 321-359.

2. Breuninger WF, Piyachomkwan K, Sriroth K. Tapioca/cassava Starch Production and Use. In: Be Miller J, et al. editors. Starch: Chemistry and Technology. 3rd ed. New York, USA: Academic Press; 2009. p. 569-578.

3. Abraham TE. Stabilization of paste viscosity of cassava by heat moisture treatment. Starch-Starke. 1993;45(4):131-135.

4. Santayanon R, Wootthikanokkhan J. Modification of cassava starch by using propionic anhydride and properties of the starch-blended polyester polyurethane. CarbohydPolym. 2003;51:17-24.

5. Jacobs H, Delcour JA. Hydrothermal modifications of granular starch, with retention of the granular structure: A review. J Agr Food Chem. 1998;46(8):2895-2905.

6. Hoover R. The impact of heat-moisture treatment on molecular structures and properties of starches isolated from different botanical sources. Crit Rev Food Sci. 2010;50(9):835-847.

7. Hoover R, Vasanthan T. Effect of heat-moisture treatment on the structure and physicochemical properties of cereal, legume, and tuber starches. Carbohyd Res. 1994;252:33-53.

8. Chung HJ, Hoover R, Liu Q. The impact of single and dual hydrothermal modifications on the molecular structure and physicochemical properties of normal corn starch. Int J Biol Macromol. 2009;44(2):203-210.

9. AOAC. Official methods of analysis. 18thed. Maryland, USA: Association of Official Analytical Communities; 2005.
10. Juliano BO. A simplified assay for milled rice amylose. Cereal Sci Today. 1971;16:334-360.

11. AACC. Approved methods of the AACC. 10th ed. St. Paul, MN, USA: American Association of Cereal Chemists; 2000

12. Collado LS, Corke H. Heat-moisture treatment of sweet potato starch. Food Chem. 1999;65:339-346.

13. Lu S, Chen CY, Lii CY. Gel-chromatography fraction and thermal characterization of rice starch affected by hydrothermal treatment. Cereal Chem. 1996;73(1):5-11.

14. Miyoshi E. Effects of heat-moisture treatment and lipids on gelatinization and retro gradation of maize and potato starches. Cereal Chem. 2002;79(1):72-77.

15. Hoover R, Manuel H. The effect of heat-moisture treatment on the structure and physicochemical properties of normal maize, waxy maize, dull waxy maize and amylomaize V starches. J Cereal Sci. 1996;23:153162 .

16. Hoover R, Manuel H. Effect of heat-moisture treatment on the structure and physicochemical properties of legume starches. Food Res Int. 1996;29:731-750

17. Gunaratne A, Hoover R. Effect of heat-moisture treatment on the structure and physicochemical properties of tuber and root starches. Carbohyd Polym. 2002;49:425-437.

18. Donovan JW, Lorenz K, Kulp K. Differential scanning calorimetry of heat-moisture treated wheat and potato starches. Cereal Chem. 1983;60(5):381-387.

19. Cooke D, Gidley MJ. Loss of crystalline and molecular order during starch gelatinization: Origin of enthalpic transition. Carbohyd Res. 1992;227:103-122.

20. Yuan RC, Thompson DB, Boyer CD. Fine structure of amylopectin in relation to gelatinization and retrogradation behavior of maize starches from three wx-containing genotypes in two inbred lines. Cereal Chem. 1993;70(1):81-89.

21. Khunae P, Tranb T, Sirivongpaisala P. Effect of heat-moisture treatment on structural and thermal properties of rice starches differing in amylose content. Starch-Starke. 2007;59(12):593-599.

22. Chung HJ, Liu Q, Hoover R. Impact of annealing and heat-moisture treatment on rapidly digestible, slowly digestible and resistant starch levels in native and gelatinized corn, pea and lentil starches. Carbohyd Polym. 2009;75:436-447.

23. Hoover R. Composition, molecular structure, and physicochemical properties of tuber and root starches: A review. Carbohyd Polym. 2001;45:253-267.

24. Englyst HN, Kingman SM, Cummings JH. Classification and measurement of nutritionally important starch fractions. Eur J ClinNutr. 1992;46 (Suppl 2):S33-S50.

25. Gallant DJ, Bouchet B, Buleon A, et al. Physical characteristics of starch granules and susceptibility to enzymatic degradation. Eur J ClinNutr. 1992;46 (Suppl 2):S3-S16.

26. Kawabata A, Takase N, Miyoshi E, et al. Microscopic observation and X-ray diffractometry of heat-moisture treated starch granules. StarchStarke. 1994;46(12):463-469.

27. Brumovsky JO, Thompson DB. Production of boiling stable granular resistant starch by partial acid hydrolysis and hydrothermal treatments of high amylose maize starch. Cereal Chem. 2001;78(6):680-689. 
28. Bhattacharya M, Zee SY, Corke H. Physicochemical properties related to quality of rice noodles. Cereal Chem. 1999;76(6):861-867.

29. Lorenz K, Kulp K. Cereal and root starch modification by heat-moisture treatment. I. Physicochemical properties. Starch-Starke. 1982;34(2):50 54.
30. Choi SG, Kerr WL. Water mobility and textural properties of native and hydroxypropylated wheat starch gels. Carbohyd Polym. 2003;51:1-8. 